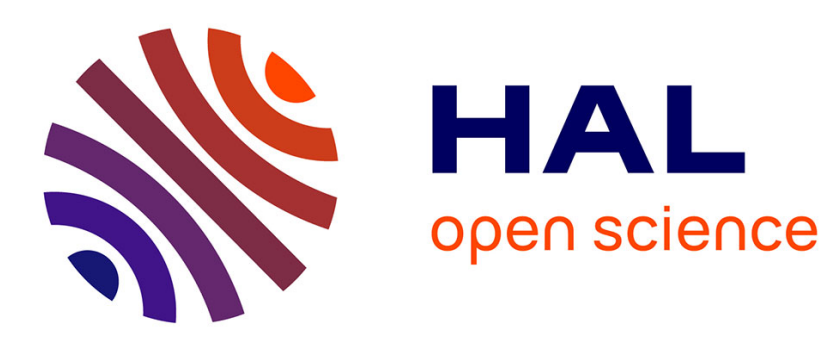

\title{
Le visage et le nom. Réflexions sur les interfaces divines à la lumière de la documentation proche-orientale
}

\author{
Corinne Bonnet
}

\section{To cite this version:}

Corinne Bonnet. Le visage et le nom. Réflexions sur les interfaces divines à la lumière de la documentation proche-orientale. Bodiou, Lydie; Mehl, Véronique; Oulhen, Jacques. Chemin faisant. Mythes, cultes et société en Grèce ancienne. Mélanges en l'honneur de Pierre Brulé, pp.205-214, 2009, 978-2-7535-0946-7. hal-01996850

\section{HAL Id: hal-01996850 \\ https://hal.science/hal-01996850}

Submitted on 20 Feb 2019

HAL is a multi-disciplinary open access archive for the deposit and dissemination of scientific research documents, whether they are published or not. The documents may come from teaching and research institutions in France or abroad, or from public or private research centers.
L'archive ouverte pluridisciplinaire HAL, est destinée au dépôt et à la diffusion de documents scientifiques de niveau recherche, publiés ou non, émanant des établissements d'enseignement et de recherche français ou étrangers, des laboratoires publics ou privés. 
Claire Sotinel et Maurice SARTRE (dir.),

L'usage du passé entre Antiquité tardive et haut Moyen Âge. Hommage à Brigitte Beaujard, 2008, $146 \mathrm{p}$.

Véronique Mehl et Pierre Brulé (dir.),

Le sacrifice antique. Vestiges, procédures et stratégies, 2008, $280 \mathrm{p}$.

Véronique Dasen et Jérôme WiLgaux (dir.),

Langages et métaphores du corps dans le monde antique, 2008, 260 p.

Dominique Lhuillier-MarTinetTi,

Lindividu dans la famille à Rome au IV siècle. D'après l'auvre d'Ambroise de Milan, 2008, 264 p.

Brigitte Le Guen (dir.),

À chacun sa tragédie? Retour sur la tragédie grecque, 2007, 172 p.

Laurent CAPDETREY,

Le pouvoir séleucide. Territoire, administration, finances d'un royaume hellénistique (312-129 av. J.-C.), 2007, 536 p.

Pierre Brulé, Jacques Oulhen et Francis Prost (dir.),

Économie et société en Grèce antique (478-88 av. J.-C.), 2007, 436 p.

Claudia de Oliveira Gomes,

La cité tyrannique. Histoire politique de la Grèce archaïque, 2007, $232 \mathrm{p}$.

Pierre BRulé,

La Grèce d'à côté. Réel et imaginaire en miroir en Grèce antique, 2007, 544 p.

Philippe Monbrun,

Les voix d'Apollon. L'arc, la lyre et les oracles, 2007,350 p.

Marie-Thérèse CAM (dir.),

La médecine vétérinaire antique. Sources écrites, archéologiques, iconographiques, 2007, 328 p.

Rita Compatangelo-Soussignan et Christian-Georges Schwentzel (dir.),

Etrangers dans la cité romaine. "Habiter une autre patrie": des incolae de la République aux peuples fédérés du Bas-Empire-», 2007, 272 p.

Yves LAFOND,

La mémoire des cités dans le Péloponnèse d'époque romaine (It siècle avant J.-C.-IIt siècle après J.-C.), 2006, 388 p.

Claudine Auliard,

La diplomatie romaine. L'autre instrument de la conquête. De la fondation à la fin des guerres samnites (753-290 av. J.-C.), 2006, $344 \mathrm{p}$

Michel Mouin (dir.),

Les régulations sociales dans l'Antiquité, 2006, 428 p.

Lydie Bodiou, Dominique Frère et Véronique Mehl avec la collaboration d'Alexandre Tourraix,

L'expression des corps. Gestes, attitudes, regards dans l'iconographie antique, 2006, 392 p.

Francis Prost et Jérôme Wilgaux (dir.),

Penser et représenter le corps dans l'Antiquité, 2006, 416 p.

Claudine Auliard et Lydie Bodiou (dir.),

Au jardin des Hespérides. Histoire, société et épigraphie des mondes anciens, 2004, $416 \mathrm{p}$.

\section{Chemin faisant}

\section{Mythes, cultes et société en Grèce ancienne}

\section{Mélanges en l'honneur de Pierre Brulé}

$$
229458
$$

Collection Histoire

Presses Universitaires de Rennes 


\section{Table des matières}

Lydie Bodiou, Véronique Mehl, Jacques Oulhen,

Francis Prost et Jérôme WiLgaux

Abécédaire inachevé. Fragments pour une biographie non autorisée.

Lydie Bodiou, Véronique Mehl, Jacques Oulhen,

Francis Prost et Jérôme Wilgaux

Introduction...

Première partie

\section{Mise en route}

Pierre BRIANT

Le thème de la "décadence perse" dans l'historiographie européenne

du XVIIf siècle: remarques préliminaires sur la genèse d'un mythe

Stéphanie MAILLOT

Une association de sculpteurs à Rhodes au If siècle av. J.-C. :

un cercle d'intégration à la société rhodienne....

Marie-Madeleine Mactoux

Esclave, fouet, rituel.

Anton Powell

Sparte: comment déchiffrer ses idéaux?

Deuxième partie

\section{Chemins de femmes}

Laurent PIOLOT

À l'ombre des maris

Louise BruIT-ZaIDMAN

Les femmes et le religieux dans les Lois de Platon

Anne JACQuemin

Pendant quà Olympie les hommes...

ou séparés pour être plus efficaces. 
Karine Karila-CoHEN

Les filles d'Athènes à Delphes: femmes, religion et société

à travers l'exemple des canéphores de la Pythaïde.

Claudine Leduc

L'énigmatique Kourotrophos et l'Olivier de l'Acropole

\section{Troisième partie \\ Vers le polythéisme}

Robert PARKer

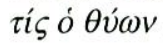

Madeleine JosT

À propos des généalogies de Pan

Paolo SCARPI

Les visages du héros, discours mythique et schéma rituel,

pour une projection panhellénique

Sylvain LeBreton

Dionysos Ômèstès (Plutarque, Thémistocle, 13 ; Antoine, 24)

Corinne Bonnet

Le visage et le nom. Réflexions sur les interfaces divines

à la lumière de la documentation proche-orientale.

Stella Georgoudi

Questions pythiques: retour sur le(s) trépied(s) et le laurier d'Apollon

\section{Quatrième partie \\ Itinéraires corporels}

Hélène BEctarTe

Le costume de l'épouse dans l'art funéraire attique de l'époque classique

Rachel Touzé

Le myrte et Aphrodite, quelque part entre le désir et le dégoût

Gaucelm Borsmenu

"La vierge sans mère":

les métamorphoses d'Athéna adolescente dans l'Odyssée.

Véronique DASEN

Une "Baubô" sur une gemme magique

Gwenaëlle Le Person

Soigner l'épilepsie (Hippocrate, Maladie sacrée). Existe-t-il une opposition entre la médecine "populaire" des magoi et la médecine "rationnelle" des Hippocratiques dans le traitement de la maladie? 


\section{Le visage et le nom \\ Réflexions sur les interfaces divines à la lumière de la documentation proche-orientale}

Corinne BonneT

Université de Toulouse 2

\section{Des épiclèses au féminin \\ dans les polythéismes grec et phénicien}

Nul, mieux que Pierre Brulé, n’a souligné l'importance des épiclèses comme opérateur logique au sein des panthéons polythéistes ${ }^{1}$. Elles irriguent le système de pensée symbolique en créant des connections ou des polarités entre les divinités en rapport avec les champs de compétence et d'action. Elles éclairent, pour nous, historiens des religions, le spectre protéiforme des fonctions divines. Si la richesse des sources grecques - littéraire, épigraphique et iconographique - permet aisément d' "expérimenter dans le champ du polythéisme ${ }^{2}$ ", l'avarice documentaire d'autres secteurs culturels peut rendre cette opération bien plus difficile. Je songe en particulier au domaine phénicien et punique qui repose sur une assise documentaire plus restreinte, privée surtout de toute trace de littérature ${ }^{3}$, donc de discours mythologique, ce qui n'est pas le moindre des paradoxes s'agissant des «inventeurs» de l'alphabet!

Parmi les dix mille inscriptions (environ) en langue phénicienne et punique conservées dans tout l'espace méditerranéen, les épiclèses ne manquent pas. Elles n'ont cependant fait l'objet d'aucun répertoire ni d'aucune étude systématique, ne serait-ce que parce que les corpus dont on dis-

1. Brulé P., «Le langage des épiclèses dans le polythéisme hellénique (l'exemple de quelques divinités féminines)", Kernos, 11, 1998, p. 13-34; Belayche N., Brulé P., Freyburger G., Lehmann Y., Pernot L., Prost F. (éd.), Nommer les Dieux. Théonymes, épithètes, épiclèses dans l'Antiquité, Turnhout - Rennes, Brepols - Presses universitaires de Rennes, 2005; Brulé P., LeBreton S., "La banque de données sur les épiclèses divines (BDDE) du Crescam: sa philoso-

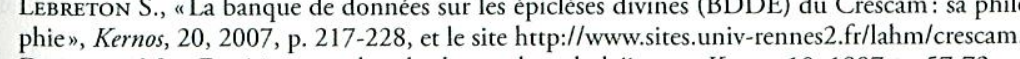

phie», Kernos, 20, 2007, p. 217-228, et le site http://www.sites.univ-rennes2.fr/lahm/crescam.
2. Detienne M., "Expérimenter dans le champ du polythéisme», Kernos, 10, 1997, p. 57-72.

3. Krings V., "La littérature phénicienne et punique», Krings V. (éd.), La civilisation phénicienne et punique. Manuel de recherche, Leiden-Boston, 1995, p. 31-38. 
pose sont aujourd'hui largement dépassés ${ }^{4}$. Depuis Renan, et son Corpus Inscriptionum Semiticarum, lancé en 1883, la documentation s'est enrichie et a été l'objet de multiples révisions. Il n'est donc pas facile d'avoir une vue d'ensemble du "système épiclétique» phénicien et punique. Posée dans ces termes, la question mérite d'être elle-même "questionnée». Car est-il légitime de penser que l'on a affaire à un système unitaire qui puisse être étudié comme tel? L'imagerie traditionnelle des Phéniciens les associe à une dimension spatiale fluctuante: marins, voyageurs, explorateurs, ils ont pour ancrage les rives de la Méditerranée, de la mythique Forêt de cèdres aux prés verdoyants d'Erythie où le soleil se couche. Sonder les désignations cultuelles des Phéniciens et des Puniques, c'est d'emblée se placer dans une perspective de religio migrans ${ }^{5}$, au sein de laquelle le rapport entre religion et territoire, que traduisent souvent les épiclèses, répond à une logique particulière, celle d'un ancrage dans les lieux que l'on investit, mais aussi dans la mémoire des lieux que l'on reproduit ${ }^{6}$. On évitera donc de raisonner en termes de système, de "religion phénico-punique» pour privilégier le bricolage identitaire de communautés à la fois sédentaires et nomades, auquel les épiclèses peuvent faire écho.

On aurait cependant tort de se focaliser exclusivement sur la dimension migratoire qui occupe, il me semble, déjà trop de place dans les études phéniciennes et puniques, au détriment des enquêtes portant sur le substrat social et symbolique des pratiques cultuelles. À cet égard, on s'est peu intéressé à la projection de l'univers féminin sur le plan divin. "Dans la distribution des genres (et des sexes) chez ces immortels grecs, la position du féminin est rien moins que subalterne», note Pierre Brulé7, une observation que l'on peut reprendre à son compte pour le domaine phénicien et punique. Il serait naïf de croire que la société divine fonctionne sur le mode spéculaire par rapport à la société humaine, ne serait-ce que parce que divers niveaux, biologiques et sociaux, s'entremêlent dans les statuts des genres qui interagissent au sein de l'une et de l'autre. Cela dit, il me semble incontestable que les royaumes phéniciens de la côte levantine ont structuré leurs panthéons sur le modèle de la structure politique dominante. Nous avons certes affaire à des panthéons polythéistes richement dotés, mais ils sont nettement dominés par le couple divin que forment un Baal

4. On dispose désormais d'un instrument de ce type pour les textes ougaritiques, dont le corpus est aisément repérable: Rahmouni A., Divine Epithets in the Ugaritic Alphabetic Texts, Leiden-Boston, 2008, qui recense 112 épiclèses. Pour la documentation akkadienne, TallQvist K., Akkadische Götter-epitheta, Helsinki, 1938.

5. Sur ce concept, voir Auffarth C., "Religio migrans. Die "Orientalischen Religionen" im Kontext antiker Religion. Ein theoretisches Model ", Bonnet C., Ribichini S., Steuernagel D. (éd.), Religioni in contatto nel Mediterraneo antico. Modalità di diffusione e processi di interferenza, Pise, 2008, p. 333-363.

6. Bonnet C., "Melqart in Occidente. Percorsi di appropriazione e di acculturazione", Bernardini P., Zucca R. (éd.), Il Mediterraneo di Herakles, Rome, 2005, p. 17-28, sur cette utilisation "mémoriale" des épiclèses.

7. BRULÉ P., Les femmes grecques à l'époque classique, Paris, 2001, p. 17. Sur ce sujet, voir aussi RUDHARDT J., "Reflets de la féminité dans le miroir de la mythologie grecque", Les dieux, le féminin, le pouvoir, Borgeaud Ph., Pirenne Delforge V. (éd.), Genève, 2006, p. 41-71. poliade et son épouse, qualifiée de Baalat («Maîtresse»), Milkat («Reine»), Rabat ("Grande»), ou plus simplement nommée Astarté ${ }^{8}$. Qu'il s'agisse de Byblos, de Sidon ou de Tyr, pour prendre les cas les mieux documentés, la configuration pyramidale du panthéon est évidente, en écho à celle de la société civile, dominée par la présence autoritaire et bienfaisante à la fois du souverain. Les rapports entre l'élément masculin et l'élément féminin, au sein de cette dyade, sont plus complexes qu'il pourrait y paraître de prime abord. Là où la religion grecque place Zeus dans une multiplicité de relations différentes, avec Héra, Aphrodite, Athéna, Hestia, etc., la religion phénicienne, qui apparaît comme le fruit d'une longue évolution des panthéons ouest-sémitiques de l'Âge du Bronze, place, face à Baal, une interlocutrice majeure, au profil complexe. Astarté, puisque c'est d'elle qu'il s'agit, n'est pas la seule déesse attestée, mais elle apparaît comme une figure fortement théocrasique ou hénothéiste qui concentre dans ses mains une panoplie considérable d'attributions, laissant dans l'ombre les Anat ou Athirat, pourtant prééminentes au Bronze Récent dans le panthéon ougaritique, notamment.

C'est avec elle que le roi - souverain divin ou roi historique - compose. Ils sont inséparables et profondément solidaires. Le roi est celui qui occupe le trône, qui guerroie, qui protège le territoire, qui construit et restaure les sanctuaires, qui "fait vivre son peuple ${ }^{9}$ ». Il est, dans ces rôles fondamentaux, l'hypostase et l'héritier des rois mythiques, fondateurs de la cité et de la dynastie. Mais cette force, cette vitalité et cette légitimité à agir pour tous, au nom des dieux, le roi la reçoit de la déesse. La femme est, biologiquement, détentrice du pouvoir extraordinaire de donner la vie Culturellement, elle investit donc le roi du charisme nécessaire à l'exercice de ses fonctions ${ }^{10}$ : les inscriptions phéniciennes, de Byblos et de Sidon en particulier, montrent que le roi tient ses pouvoirs de la déesse, qui l'a choisi, qui "prolonge ses jours", qui fait de lui un monarque juste et prospère ${ }^{11}$. Sur le plan mythique, cette forte interdépendance s'exprime par le biais de divers récits relatifs à une série, assez hétérogène de divinités qui font l'expérience de la mort ou, pour le moins, d'une disparition, suivie d'un retour à la vie ou d'une réapparition, comme Adonis à Byblos ${ }^{12}$. Dans chaque cas, l'élément féminin joue un rôle actif et décisif dans les vicissitudes du dieu: détentrice de vie, la déesse l'insuffle au dieu mourant pour le ramener à la vie et à l'efficience. C'est un moment crucial qui symbolise rituellement

8. Sur cette déesse, je renvoie à ma monographie Bonnet C., Astarté. Dossier documentaire et perspectives historiques, Rome, 1996

9. Xella P., "Il re», Zamora J.A. (éd.), El hombre fenicio. Estudios y materiales, Rome, 2003, p. 23-32.

10. BonNET C., "La déesse et le roi : nouveaux regards sur le rôle d'Astarté dans les rites d'investiture en Phénicie", Mythos n.s. 1, 2008, p. 11-23.

11. Bonnet C., Astarté, op. cit., passim.

12. Mettinger T.N.D., The Riddle of Resurrection: "Dying and Rising Gods" in the ancient Near East, Stockholm, 2001; Xella P. (éd.), Quando un dio muore, Vérone, 2001. 
le renouvellement annuel des énergies cosmiques, biologiques et sociales à l'œuvre dans chaque communauté.

Les épiclèses de la déesse traduisent, à leur façon, synthétique et allusive, cette articulation complexe. Ils constituent des énoncés théologiques minimalistes pourtant très éclairants. Astarté est donc principalement la "grande», la "reine», la "maîtresse». Ces épiclèses récurrentes renvoient, on le comprend, à un arrière-plan théologique implicite que l'absence de mythologie - sinon par le biais des échos qu'en donne la mythologie grecque - ne permet pas de déployer, comme on le fait habituellement dans l'étude des panthéons grecs. Deux épiclèses, beaucoup plus rares, vont néanmoins nous permettre d'approfondir quelque peu l'analyse des rapports entre le dieu et la déesse, entre le masculin et le féminin, entre le panthéon et la société, dans un jeu de va-et-vient qui n'est cependant jamais mécanique.

\section{Connaître le nom du dieu}

Dans la longue inscription du sarcophage d'Eshmunazor ${ }^{13}$, roi de Sidon aux alentours de 500 av. J.-C., le jeune souverain défunt relate les faits marquants de son règne, en particulier les nombreuses marques de dévotion envers ses protecteurs divins, Astarté, Eshmun et le Baal de Sidon, ce dernier étant, à mon sens, une désignation d'Eshmun. Eshmunazor est un roi-bâtisseur, pieux et fidèle serviteur des dieux et de son suzerain perse, qui se met en scène. Diverses zones du territoire sidonien sont concernées par les activités du roi ; elles ont fait l'objet de discussions nombreuses ${ }^{14}$, mais ce qui me semble se dégager d'une relecture attentive de ce texte pourtant très connu, c'est une répartition significative des noms et des épiclèses en fonction des configurations topiques et fonctionnelles:

1. un temple pour Astarté dans Sidon-de-la-mer;

2. un temple pour Eshmun, le "prince saint", à la source Ydal, dans la montagne;

3. deux temples aux dieux de Sidon dans Sidon-de-la-mer, à savoir le Baal de Sidon et Astarté «Nom de Baal» (šm bl).

Dans le premier lieu de culte, où elle est vénérée seule, Astarté ne reçoit pas d'épiclèse; en revanche, dans le second sanctuaire, où elle fait partie d'un couple divin - chacun possédant son propre bt ("temple») - Astarté et son parèdre reçoivent des noms corrélés: Eshmun ${ }^{15}$ est le Baal poliade et Astarté est associée à lui en tant que "Nom » du dieu. Le couple semble soudé au sein d'une relation d'interdépendance que les épiclèses et les titres traduisent habilement, tout comme la géographie cultuelle.

\section{KAI 14 .}

14. Cf. en dernier lieu avec toute la bibliographie, ZAMORA J.A., "The inscription from the first year of King Bodashtart of Sidon's reign : CIS I, 4", Orientalia, 76, 2007 (= Studi in onore di M.G. Amadasi), p. 100-113.

15. Il s'agit, à mon sens, bien de lui, dans sa fonction poliade.
Pour mieux comprendre le sens de cette épiclèse "Nom de Baal ${ }^{16}$ ", il faut en chercher d'autres traces; elles sont rares pourtant. On la rencontre dans les textes ugaritiques du Bronze Récent et peut-être dans un graffito phénicien d'Abydos ( $\mathrm{V}^{\mathrm{e}}$-III ${ }^{\mathrm{e}}$ siècle av. J.-C.) ${ }^{17}$. Les deux textes ugaritiques qui font allusion à Atthart "Nom de Baal» renferment une même formule de malédiction dans laquelle la déesse, en compagnie du dieu serpentiforme Horon, intervient pour anéantir un ennemi ${ }^{18}$ : «Puisse Horon écraser ta tête, Astarté "Nom de Baal", ton crâne.»

Les contextes méritent notre attention. Dans le récit de la lutte entre Baal et Yam (l'élément aquatique chaotique), cette invocation menaçante est lancée par Baal contre son ennemi ${ }^{19}$, tandis que, dans le mythe de Keret, elle figure au terme de la $4^{\mathrm{e}}$ colonne de la $3^{\mathrm{e}}$ tablette et est prononcée par le roi contre son fils Yassibu qui, une fois la guérison du roi acquise, conçoit le projet de le renverser. Les ugaritologues discutent sur le point de savoir si ce passage clôt le récit (un colophon figure à la suite, mais il pourrait signer une tablette intermédiaire) ou le fait, en quelque sorte, rebondir vers d'autres développements. Peu importe ici. Nous retenons de ces deux passages que l'épiclèse "Nom de Baal» appliquée à la déesse Astarté affiche une valeur performative très forte. Elle accompagne la déesse en train d'agir puissamment: elle fracasse la tête de ses ennemis, menace ceux qui mettent en péril l'ordre cosmique ou social, la justice dont les dieux sont les garants.

Or, on connait, par ailleurs, une manifestation de Baal, sous le nom de Baal șmd, c'est-à-dire "Baal de la massue», qui est attesté dans la malédiction finale de l'inscription phénicienne du roi Kilamuwa, à Zinjirli ${ }^{20}$, en secteur araméen, au $\mathrm{IX}^{\mathrm{e}}$ siècle av. J.-C.: le dieu a pareillement pour mission de détruire les personnes qui porteraient atteinte à l'inscription, donc à "l'ordre public». Ce dieu violent, qui brandit une massue, est certainement le Smiting God, le dieu en puissance largement attesté dans l'iconographie syro-palestinienne ${ }^{21}$, dont il existe du reste une version féminine, la Smiting Goddess ${ }^{22}$.

16. Cf. quelques éléments rapides et superficiels d'interprétation dans VAN DER ToorN K. BeCKING B., VAn Der Horst P. (éd.), Dictionary of Deities and Demons in the Bible, $2^{e}$ éd., Leiden, 1999 , p. 110.

17. Bonnet C., op. cit., p. 65 et 141. Bizarrement, Rahmouni A., op. cit., ne prend pas cette épiclèse en compte.

18. KTU 1.2 I 8 et 1.16 VI 56. Sur Horon, cf. VAn der Toorn K., Becking B., Van der Horst P. (éd.), op. cit., p. 425-426. Je me demande s'il ne faut pas chercher du côté de modèles égyptien pour expliquer son rôle de dieu destructeur ici. Voir aussi, pour son rôle dans la première incantation d'Arslan Tash (en compagnie de ses "huit femmes»), ConkLin B.W., "Arslan Tash I and other vestiges of a particular Syrian incantatory thread ", Biblica, 84, 2003, p. 89-101.

19. La localisation de ce passage au sein de l'ensemble du récit, en raison de l'état lacunaire des

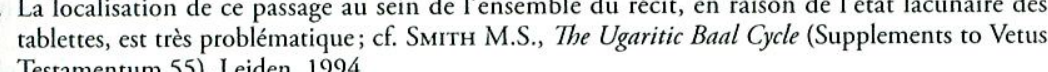

20. KAI 24. Tropper J., Die Inschriften von Zincirli, Münster, 1993, p. 20-21, 45.

21. Cornelius I., The iconography of the Canaanite God's Reshef and Ba'al. Late Bronze and Iron Age I Periods (c. 1500-1000 BCE), Fribourg-Göttingen, 1994.

22. Cornelius I., The many faces of the goddess. The iconography of the Syro-Palestinian goddess Anat Astarte, Qedeshet, and Asherah, c. 1500-1000 BC, Fribourg - Göttingen, 2004. 
On semble autorisé à déduire de ces rapprochements que, lorsqu'elle se présente comme le bras armé de Baal, Astarté agit «en son nom » : elle est le "Nom de Baal». Cette épiclèse révèle l'existence d'un lien puissant et actif entre le dieu et la déesse. C'est pourquoi, dans l'inscription d'Eshmunazor, à Sidon, lorsque le Baal poliade œuvre avec Astarté pour défendre la cité et promouvoir son bien-être, celle-ci apparaît sous la forme de la déesse «Nom de Baal».

À ce stade, il reste à mettre en lumière la manière dont le "Nom " peut se transformer en énergie performante, en puissance active. Quelles représentations du divin se profilent derrière ces titres? Un bref extrait d'Origène peut nous mettre sur la piste:

"Après cela, il (= Celse) dit que "ces gardeurs de chèvres et de moutons ont cru qu'il y a un seul dieu, Très-Haut, ou bien Adonaïos, ou bien Celeste, ou bien Sabaoth, ou quel qu'autre nom encore qu'ils se plaisent à donner à ce monde, mais qu'ils n'ont rien su de plus". Et dans ce qui suit, il affirme que "cela ne change rien d'appeler le dieu suprême Zeus, du nom qu'il porte chez les Grecs, ou Untel, pour ainsi dire, chez les Indiens, ou Untel chez les Égyptiens".

On doit dire en réponse aussi à cela que ce sujet touche à la théorie profonde et ineffable de la nature de noms: est-ce que, comme le pense Aristote, les noms existent par convention, ou bien, comme l'estiment les stoïciens, existent-ils par nature, parce que les sons premiers représentent les choses sur lesquelles s'appliquent les noms, en fonction de quoi les stoïciens proposent des éléments de base d'étymologie, ou bien, comme l'enseigne Épicure, à la différence de ce que pensent les stoïciens, existent-ils par nature, parce que les premiers hommes ont fait jaillir certains sons qui s'appliquent aux choses ${ }^{23}$ ?»

Ce texte - mais de nombreux autres vont dans le même sens ${ }^{24}$ - montre que le théonyme est au cœur de la réflexion théologique et de la pratique cultuelle. Il dit le dieu, il révèle sa nature et ses pouvoirs. C'est pourquoi, dans le monothéisme hébraïque, il ne peut être prononcé ${ }^{25}$. En domaine phénicien et punique, on connaît du reste un dieu Shem, "Nom», qui entre en composition dans divers anthroponymes théophores, comme Shemy ou Shembaal ${ }^{26}$. Celui qui connaît le nom d'une divinité peut exercer sur elle un pouvoir contraignant, l'activer, la rendre présente et efficace. De fait, tous les rituels commencent par l'invocation de la divinité: on prononce son nom pour susciter son épiphanie agissante. Astarté, en tant que «Nom de Baal», incarne donc la puissance performative du dieu, dans le cadre

23. Origène, Contre Celse, I, 24-25. Pour toutes les facettes de cette «théologie du nom ", voir Belayche N., Brulé P., Freyburger G., Lehmann Y., Pernot L., Prost F. (éd.), op. cit.

24. Comment oublier Socrate auquel Platon fait dire, dans le Philèbe (12c): «J'ai toujours eu pour les noms des dieux [...] une révérence plus qu'humaine, une crainte qui passe toute limite" (trad. A. Diès, CUF).

25. MetTinger T.N.D., The Dethronement of Sabaoth. Studies in the Shem and Kabod Theologies, Uppsala, 1982

26. Cf. VAn der Toorn K., Becking B., Van der Horst P. (éd.), op. cit., p. 763; Lubetski M., « ¿̌m as a deity", Religion, 17, 1987, p. 1-14 (qui interprète erronément "Astarté Shem Baal " comme une triade); BonNET C., "Antipatros l'Ascalonite dévoré par un lion. Commentaire à CIS I, 115 ", Mélanges M. Sznycer (= Semitica 38, 1990), p. 39-47. d'un rapport étroit et privilégié; du reste, c'est précisément dans une formule incantatoire que cette Erscheinungsform de la déesse apparaît à deux reprises.

Le "Nom», par sa puissance opérante, concourt ainsi au "Renom» du dieu puisqu'il favorise l'accomplissement d'exploits ${ }^{27}$. On se rapproche ici de la notion grecque de kleos, si richement mise en scène dans l'épopée homérique. Et comment ne pas songer à «celui qui tire son nom/renom d'Héra ", Héraclès, le héros des innombrables travaux ${ }^{28}$. Les fidèles, qui, eux, ignorent le véritable Nom du dieu, sa véritable nature, son "essence", ont la possibilité de s'adresser à la déesse afin qu'elle opère une intercession. La déesse sert en somme d' "interface» avec le dieu. D'ailleurs, dans l'épitaphe d'Eshmunazor examinée ci-dessus, le dieu associé à Astarté n'est-il pas simplement désigné comme le Baal de Sidon associé à la déesse, sans que son nom soit précisé?

\section{Contempler le visage du dieu}

Qu'il s'agisse du polythéisme ou du monothéisme, la connaissance des dieux ou de Dieu par les hommes est rarement immédiate, s'agissant de deux univers ontologiquement et soigneusement séparés. Le culte a dès lors pour vocation de tracer une voie de médiation entre les deux sphères, de mettre en œuvre des stratégies de communication. Le recours aux formules, aux invocations, aux noms et épiclèses, à la parole efficiente est une de celles-ci. Mais, on peut également activer un autre sens pour appréhender les dieux, à savoir la vue. Contempler les dieux directement est une expérience mystique réservée à quelques-uns ${ }^{29}$. Par l'entremise des statues, qui incarnent les dieux et présentifient leur épiphanie, nombreux sont les fidèles qui communiquent visuellement avec la sphère divine. Pour les autorités religieuses d'Israël, représenter Dieu était tout aussi inacceptable que de prononcer son nom. Le visage et le nom étaient considérés comme deux éléments constitutifs de la nature divine destinés à rester secrets. Lidolâtrie est, par exemple, clairement condamnée dans l'Exode lorsque Dieu confie à Moïse ses commandements :

"Alors Dieu prononça toutes ces paroles, en disant:

Je suis l'Éternel, ton Dieu, qui t'ai fait sortir du pays d'Égypte, de la maison de servitude.

Tu n'auras pas d'autres dieux devant ma face.

27. Les stèles commémoratives de victoires, en domaine mésopotamien - ce qu'on appelle narû - soulignent le fait que l'exploit militaire rend le nom éternellement glorieux. Voir, par exemple, l'analyse de la stèle de Dadusha, roi d'Eshnunna, qui date de 1790-1780 av. J.-C., par BARAHNI Z., Rituals of War. The Body and Violence in Mesopotamia, New York, 2008, p. 133-137.

28. Le rapport Baal-Astarté, pour ce qui est de l'efficience du nom/renom est, dans ce cas au moins, inversé. Je ne m'aventurerai certes pas dans une comparaison génétique, même si le rapprochement entre Héraclès et le type du Smiting God est tout à fait fondé. Cf. Jourdain-ANNEQuin C. Héraclès-Melqart à Amrith. Recherches iconographiques, Paris, 1992.

29. Dunand F., Boesprlug F. (éd.), Voir les Dieux, voir Dieu, Strasbourg, 2002. 
Tu ne te feras point d'image taillée, ni de représentation quelconque des choses qui sont en haut dans les cieux, qui sont en bas sur la terre, et qui sont dans les eaux plus bas que la terre.

Tu ne te prosterneras point devant elles, et tu ne les serviras point; car moi, l'Éternel, ton Dieu, je suis un Dieu jaloux, qui punis l'iniquité de pères sur les enfants jusqu'à la troisième et la quatrième génération de ceux qui me haïssent,

et qui fais miséricorde jusqu'en mille générations à ceux qui m’aiment et qui gardent mes commandements.

Tu ne prendras point le nom de l'Éternel, ton Dieu, en vain ; car l'Éternel ne laissera point impuni celui qui prendra son nom en vain ${ }^{30}$."

Dans le domaine phénicien et punique, même si l'on a depuis longtemps relevé des tendances aniconiques, l'anthropomorphisme divin est largement attesté ${ }^{31}$. Les dieux ont un visage que les fidèles peuvent observer sur une multiplicité de supports: statues, reliefs, stèles, monnaies, sceaux, etc. En vérité, ce sont des images que les fidèles regardent, et pas directement le corps divin. Mais il est une déesse qui bénéficie d'un accès privilégié au vrai visage de Baal, donc à sa personnalité, à sa volonté, à ses desseins: c'est Tanit ${ }^{32}$.

Sur des milliers de stèles provenant des tophet nord-africains, Tanit est, en effet, pn bl, «face de Baal». Ce n'est pas la seule épiclèse qu'elle reçoit, puisqu'elle est aussi occasionnellement "mère" ou "génie", par exemple, mais c'est de loin la plus répandue. Elle lui colle à la peau, pourrait-on dire. Il n'est pas question d'entrer ici dans le détail des nuances, pourtant très significatives, qui distinguent les différents usages rituels attestés dans les tophet d'Afrique du Nord et d'ailleurs, pas plus que dans le débat relatif à l'existence ou non de sacrifices humains. Pour faire bref, je dirai simplement que cette existence, à mon sens, n'est pas douteuse, mais que, selon qu'on est à Carthage ou en dehors, les pratiques variaient sans doute considérablement dans leurs modalités et proportions. De surcroît, l'équation tophet $=$ sacrifices humains me parait aujourd'hui réductrice : ce sanctuaire abritait, j'en suis convaincue, un éventail de rites bien plus large qu'on ne le concevait jadis.

30. Exode 20,1-7. Sur l'aniconisme biblique et proche-oriental, voir MetTinger T.N.D., No graven image. Israelite aniconism in its ancient Near Eastern context, Stockholm, 1995; BERLEJUNG A., Die Theologie der Bilder, Fribourg-Göttingen, 1998. Pour une réflexion sur l'aniconisme en milieu grec, Cusumano N., "Polivalenze funzionali e figurative. Osservazioni su Zeus Meilichios», Mètis, n.s. 4, 2006, p. 165-192.

31. Ribichini S., "Qualche osservazione sull'antropomorfismo delle divinità fenicie e puniche", Semitica, 39, 1990, p. 127-133

32. Cf. Dictionnaire de la civilisation phénicienne et punique, Turnhout, 1992, p. 438-439. La synthèse la plus complète reste celle de HvidBerg-Hansen F. O., La déesse TNT, 2 vol., Copenhague, 1979. Voir aussi Lipinski E., Dieux et déesses de l'univers phénicien et punique, Leuven, 1995, p. 199-21 (avec des interprétations très personnelles qu’on hésitera à suivre); MüLLER H.-P. "Philologische und religionseschichtliche Beobachtungen zur Göttin Tinnit", Rivista di studi fenici, 31, 2003, p. 123-138. Sur son interpretatio rom

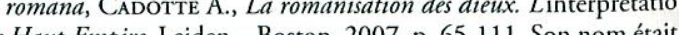
romana en Afrigue du Nord sous le Haut-Empire, Leiden-Boston, 2007, p. 65-111. Son nom était très certainement prononcé Tinnit.
Reste néanmoins que les patrons incontestés de cet espace cultuel très particulier sont Baal Hammon ${ }^{33}$ et Tanit. Au tophet de Salammbô, à Carthage, alors que le sanctuaire remonte aux origines mêmes de la ville,

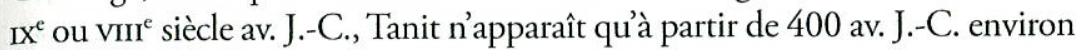
Mais, à partir de ce moment, elle précède systématiquement Baal Hammon et est accompagnée de son épiclèse traditionnelle de "Face de Baal».

On n'a guère d'éléments solides pour étayer une quelconque interprétation de ce changement significatif dans les stratégies dévotionnelles. Pourquoi les fidèles choisissent-ils alors de destiner leurs offrandes et leurs prières non plus directement au dieu, représenté dans l'iconographie comme un dieu âgé, en trône, sage et autoritaire, mais par l'intermédiaire de celle qui fait fonction d'interface? Où s'enracine ce besoin d'une intercession féminine?

Il me semble, à ce stade, pertinent de rapprocher Astarté «Nom de Baal» de Tanit "Face de Baal». La connaissance intime du dieu, de son nom ou de son visage, confere à la déesse des pouvoirs tout à fait spéciaux qu'elle met au service de son partenaire, d'une part, et des fidèles, d'autre part. Les sacrifices, sanglants ou non, pratiqués au tophet sont toujours accompagnés d'une requête ou d'un remerciement (pour grâce obtenue). La déesse, en se plaçant "face au dieu", transmet efficacement la prière des dévots, de la même façon qu'Astarté, "au nom du dieu», punit ceux qui méritent de l'être.

\section{Considérations conclusives : à la cour des puissants...}

Imbriqués et redondants comme ils apparaissent au premier regard, les panthéons s'organisent en fait en réseaux complexes de connivences et de polarités positives ou négatives; ils sont tout sauf un magma informe. Pénétrer la logique de leur organisation et de leur fonctionnement est un défi posé à nos esprits imprégnés d'un système de pensée radicalement autre. De cette modeste promenade en paysage phénicien et punique, a émergé la figure d'une divinité d' «interface», qui assume la fonction de l'intercesseur divin. Celle-ci est rendue possible par une relation particulièrement intime entre deux divinités, au sein d'un couple, source de puissance et d'efficacité.

Dans les cultures du Proche-Orient ancien, en Mésopotamie comme au Levant, c'est la matrice royale, si essentielle dans l'organisation pratique et idéologique, qui sert de modèle à la pensée religieuse; le dieu est pensé comme le roi (et vice versa); le panthéon est imaginé comme une cour; le sanctuaire est une sorte de palais ${ }^{34}$. L'intercession que nous venons de

33. Xella P., Baal Hammon. Recherches sur l'identité et l'histoire d'un dieu phénico-punique, Rome, 1991.

34. Bonnet C., "Dove vivono gli dei? Note sulla terminologia fenicio-punica dei luoghi di culto e sui modi di rappresentazione del mondo divino ", RiBichini S. et alii (éd.), Saturnia tellus, Rome, 2008, p. 673-685. 
rencontrer répond parfaitement à ce schéma. Dans les cours royales, il est rare que l'on s'adresse directement au roi et, si l'on est amené à le faire, on évite de prononcer son nom, on prend une série de précautions rhétoriques et on déclame une série de formules protocolaires, de titres ronflants visant à le glorifier et à se placer dans une position de subordination manifeste ${ }^{35}$. Il faut, pour entrer en présence du monarque, montrer patte blanche, franchir des portails et des vestibules, passer par mille et un intermédiaires pour enfin se jeter à ses pieds, sans oser contempler son visage, sans se risquer à lui adresser la parole. Il en va de même pour s'approcher des dieux. Le roi n'agit pas davantage en première ligne, mais il délègue: il a des bras armés, des agents, des fondés de pouvoir ; il est aussi entouré de femmes - la reine, la reine-mère, les favorites, les princesses - qui peuvent s'insérer dans les jeux de pouvoir et les intrigues, intercéder, favoriser, encourager. Du reste, l'inscription du sarcophage d'Eshmunazor, où apparaît Astarté «Nom de Baal ", met précisément en scène, aux côtés du roi, la puissante reine-mère Ummiashtart (nomen omen, puisque son nom signifie "Astarté est ma mère»!). Ainsi, le dieu, comme le roi, agit-il volontiers par l'intermédiaire de ses proches, «ministres" ou "épouses». Les épiclèses, dont nous sommes partis, soulignent discrètement, mais efficacement un tel fonctionnement et ses présupposés théologiques.

35. Par exemple, dans le style épistolaire d'el-Amarna où les vassaux du pharaon s'adressent à lui en ces termes: "X dit à son Seigneur, roi de (toutes les) terres, grand roi [...]. Aux pieds de mon Seigneur, mon Soleil, sept et sept fois je me jette. " Cf. Liverani M., Le lettere di el-Amarna, I. Le lettere dei "Piccoli Re", Brescia, 1998, passim. 